Article

\title{
Design of Nonequiprobable High-Order Constellations over Non-Linear Satellite Channels
}

\author{
Weigang Chen *D, Yu Peng, Changcai Han and Jinsheng Yang \\ School of Microelectronics, Tianjin University, Tianjin 300072, China; py312@tju.edu.cn (Y.P.); \\ cchan@tju.edu.cn (C.H.); jsyang@tju.edu.cn (J.Y.) \\ * Correspondence: chenwg@tju.edu.cn; Tel.:+86-138-2121-0869
}

Received: 11 December 2019; Accepted: 3 January 2020; Published: 8 January 2020

\begin{abstract}
High-order modulations are essential to improve the bandwidth efficiency of communication systems. However, such modulated signals with large envelopes are typically sensitive to the non-linear distortion caused by high power amplifiers (HPAs) in the transponder of satellite channels. In this paper, a new nonequiprobable constellation is designed to combat the non-linear effects of HPAs. We use non-uniformly distributed symbols to construct the appropriate high-order constellation for non-linear satellite channels. The nonequiprobable symbols are generated using the non-linear mapping method, which specifically consists of expansion mapping, symbol decomposition, permutation, and combination. Moreover, the demapping method adapting to the designed nonequiprobable constellation is also discussed. The simulation results show that the proposed scheme has considerable performance improvements compared with the traditional equiprobable constellations over the non-linear satellite channel.
\end{abstract}

Keywords: non-linear satellite channels; high-order modulation; nonequiprobable constellation

\section{Introduction}

As the demand for data volume and spectral efficiency in satellite communications is increasing steadily, the use of high-order modulations has become quite essential [1-3]. Considering the power limitation of the satellite, the high power amplifier (HPA) in the transponder of satellite channels often operates near the saturation point to improve the power efficiency $[4,5]$. However, due to the non-linear transfer characteristics of the HPA, non-linear distortion is introduced in the form of constellation warping and clustering at the receiver when high-order modulated signals with large envelopes are used for transmission [6-8].

Constellation design is an important approach to overcome the impact of non-linear distortion in satellite communications [9-11]. There have been numerous geometric and probabilistic proposals for the constellation optimization in the literature. The geometric proposals include devising the constellation shape [12], the position, and the separation of the constellation points $[13,14]$. However, these methods have high complexity in designing specific high-order constellations. The probabilistic proposals refer to modifying the probability of different constellation points. It has been proven theoretically that the optimal probability distribution can effectively approach the Shannon capacity limit [15-17]. The nonequiprobable constellation can be generated using signal superposition [18], or by combining forward error correction (FEC) with shaping, i.e., shaping before or after FEC. A distribution matcher is concatenated with a systematic FEC encoder in [19] for probabilistic shaping. The approaches in [20-22] are all examples of the shaping after FEC. The authors in $[20,21]$ used the many-to-one mapping to generate nonequiprobable constellations, where different junks of bits are mapped to the same constellation point. However, these schemes are known to perform poorly, as the introduced ambiguity has to be compensated by more complicated processing at the 
receiver, e.g., iterative demapping and decoding. The authors in [22] designed a shaping encoder after FEC which outputs ' 0 ' more frequently than ' 1 ', and mapped some of the bits directly to achieve a nonequiprobable distribution. However, this scheme still requires the complicated iterative demapping and decoding among the demodulator, shaping decoder, and the decoder.

In this paper, we propose a new high-order nonequiprobable constellation design method, which is particularly suitable for the non-linear satellite channel. We also use the non-uniformly distributed symbols and one-to-one mapping to generate the nonequiprobable constellation. Different from the shaping method of constructing a codebook with lowest possible Hamming weight to produce ' 0 ' more frequently than ' 1 ' in [22], we propose a non-linear mapping method to generate a much larger symbol set with multiple probabilities and further perform the permutation and combination to obtain more diverse probabilistic mapping symbols to match the constellation points with different amplitudes. The proposed scheme does not need to iterate between the decoder and demodulator. We design a non-linear mapping method to generate nonequiprobable symbol outputs and the constellation at the transmitter. Then, we provide a demapping method for the designed nonequiprobable constellation at the receiver. The simulation results demonstrate that the proposed scheme can provide the performance gain in terms of signal-to-noise ratio (SNR) efficiency over the non-linear channel.

The rest of this paper is organized as follows. Section 2 describes the system model using the proposed nonequiprobable constellation over the non-linear channel. Section 3 introduces the designed nonequiprobable high-order constellation and the non-linear mapping method for generating the nonequiprobable symbols. Section 4 gives the demapping method adapting to the proposed nonequiprobable constellation. Section 5 presents the simulation results and analysis. Finally, Section 6 concludes the paper.

\section{System Model}

Figure 1 illustrates the communication system model using the proposed nonequiprobable constellation over the non-linear channel. First, the uniformly distributed information bits/symbols are encoded at the transmitter. Then, the codewords are extendedly mapped and further processed into the nonequiprobable symbols. Finally, these symbols are mapped to generate the nonequiprobable constellation.

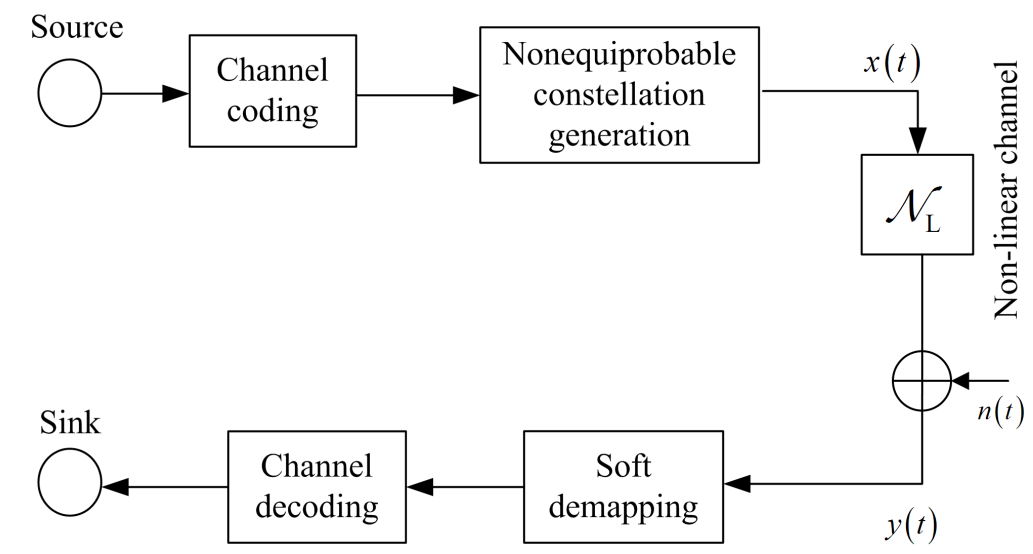

Figure 1. The system model using the proposed nonequiprobable constellation over non-linear channels.

The non-linear channel $\mathcal{N}_{\mathrm{L}}$ consists of the concatenation of the transmit filter, the non-linear amplifier, and the receive filter. Both the transmitter and receiver use the identical matched square root raised cosine (SRRC) filters [23,24]. Assume that the transmitted signal is narrow-band relative to that of the satellite transponder and the coherent detection is perfect [25]. The non-linear effect is described by the Saleh model with the amplitude-amplitude (AM/AM) and amplitude-phase (AM/PM) conversions [26]. The input is represented as 


$$
x(t)=r(t) \cdot \exp [j \phi(t)]
$$

and the output of the non-linear channel can be represented as

$$
y(t)=A[r(t)] \cdot \exp [j \phi(t)+\Phi[r(t)]]+n(t),
$$

where $y(t)$ is the received signal, $r(t)$ and $\phi(t)$ are the amplitude and phase of the modulated signal, respectively, $n(t)$ is the complex-valued additive white Gaussian noise (AWGN) with zero mean and variance $N_{0} / 2$ per dimension $[27,28] . A[r(t)]$ and $\Phi[r(t)]$ are the AM/AM and AM/PM conversions of the non-linear model, expressed as Equations (3) and (4), respectively [26].

$$
\begin{aligned}
A[r(t)] & =\frac{\alpha_{a} r(t)}{1+\beta_{a}[r(t)]^{2}}, \\
\Phi[r(t)] & =\frac{\alpha_{\phi}[r(t)]^{2}}{1+\beta_{\phi}[r(t)]^{2}},
\end{aligned}
$$

and the parameters are set to $\alpha_{a}=2, \beta_{a}=1, \alpha_{\phi}=\pi / 3, \beta_{\phi}=1[20,29]$.

At the receiver, the signal $y(t)$ is further processed by the soft demapping method to calculate the likelihood of each symbol. Finally, these soft metrics are sent to the decoder to recover the original information $[30,31]$.

\section{Nonequiprobable Constellations for Non-Linear Satellite Channels}

In this section, we take the 64-level quadrature amplitude modulation (QAM) as an example to design and generate the nonequiprobable high-order constellation suitable for non-linear satellite channels. The nonequiprobable constellation is designed according to the non-linear distortion characteristics of the signal points [20,32]. The non-linear mapping method is also proposed to generate nonequiprobable mapping symbols, which specifically consist of expansion mapping, symbol decomposition, permutation, and combination. The proposed non-linear mapping method can convert the uniformly distributed information bit/symbol inputs into the non-uniformly distributed mapping symbol outputs, thereby constructing the appropriate nonequiprobable constellation.

\subsection{Design of Nonequiprobable High-Order Constellations}

We first design the constellation $S$ and the probability $P\left(s_{l}\right)$ of signal points $s_{l} \in S$ for $l=0,1, \cdots, 63$ over the non-linear channel. Figure 2 illustrates the AM/AM and AM/PM conversions of the 64-QAM constellation at different input back off (IBO) values. It can be seen that for a high IBO value, the amplifier works near the linear region and the distorted constellation points are close to the original points. When the IBO decreases, the system works in the non-linear region and the constellation points are squeezed together, which would result in very serious non-linear distortion. In addition, it can also be seen from Equations (3) and (4) that the AM/AM and AM/PM conversions are mainly related to the amplitude $[33,34]$. Therefore, different probabilities can be designed for signal points with different amplitudes to achieve the non-uniform probability distribution of the constellation.

According to the analysis of the non-linear distortion characteristics of the equiprobable constellation, the design principles of the nonequiprobable constellation are listed as follows.

1. Signals with large amplitudes should be used less frequently.

2. Signals with small amplitudes should be used appropriately frequently.

Principles 1 and 2 are both considerations for constellation shaping gain over non-linear channels. If principle 1 is satisfied, the average signal energy will be reduced. Hence, the nonequiprobable constellation suffers from less distortion than the equiprobable one. In addition, all the outer points 
are squeezed together for both cases, but the distance among the inner points of the nonequiprobable case is larger than that of the equiprobable one [20], so that principle 2 helps to demap more correctly. Therefore, a nonequiprobable constellation designed in this way can be expected to achieve better overall performance.

The in-phase component $I$ and the quadrature component $Q$ of the 64-QAM constellation, which satisfy $I, Q \in\{ \pm 1, \pm 3, \pm 5, \pm 7\}$, constitute a total of nine amplitudes. If the amplitude is expressed as $A$, then $A \in\{\sqrt{2}, \sqrt{10}, \sqrt{18}, \sqrt{26}, \sqrt{34}, \sqrt{50}, \sqrt{58}, \sqrt{74}, \sqrt{98}\}$. Considering the above principles, we give a design example of the non-uniform probability distribution shown in Table 1, i.e., the probability and the number of signal points with different amplitudes.

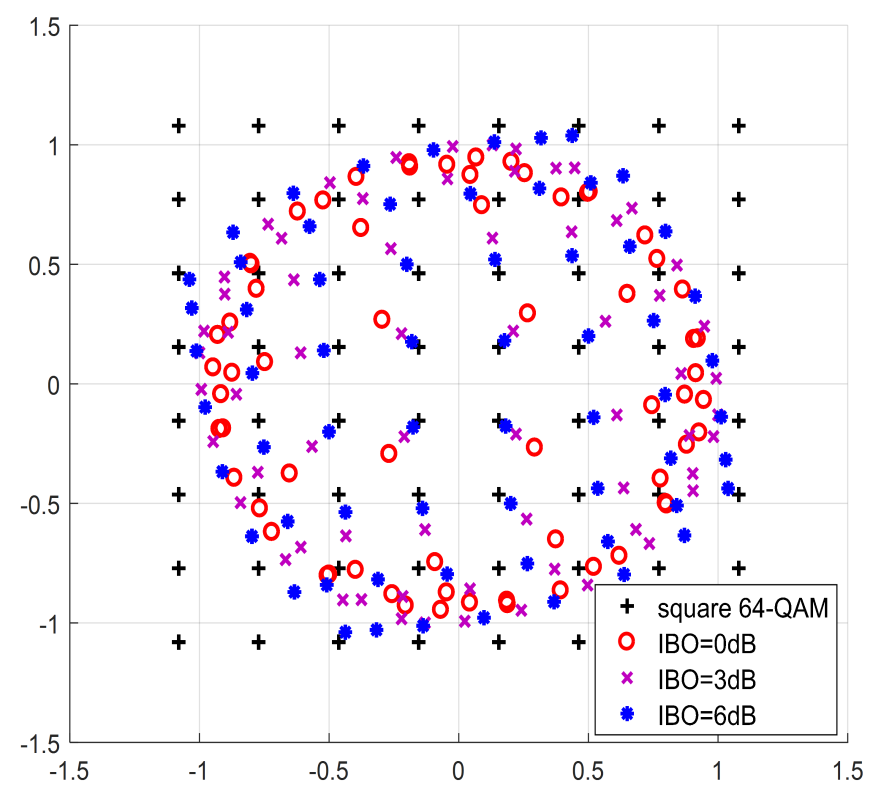

Figure 2. AM/AM and AM/PM conversions of 64-QAM equiprobable constellation at different input back off (IBO) values.

Table 1. Probability of signal points with different amplitudes.

\begin{tabular}{ccc}
\hline Amplitude & Number & Probability \\
\hline$\sqrt{2}$ & 4 & $1 / 32$ \\
$\sqrt{10}$ & 8 & $1 / 32$ \\
$\sqrt{18}$ & 4 & $1 / 32$ \\
$\sqrt{2} 6$ & 8 & $3 / 128$ \\
$\sqrt{3} 4$ & 8 & $1 / 64$ \\
$\sqrt{50}$ & 12 & $1 / 96$ \\
$\sqrt{5} 8$ & 8 & $1 / 128$ \\
$\sqrt{74}$ & 8 & 0 \\
$\sqrt{9} 8$ & 4 & 0 \\
\hline
\end{tabular}

\subsection{Nonequiprobable Symbols Adapt to the Nonequiprobable Constellation}

In order to generate nonequiprobable symbol outputs approximately distributed with the desired distribution in Table 1, we proposed the non-linear mapping method. The proposed scheme is illustrated in Figure 3.

First, the expansion mapping is performed to make the probability distribution of the elements ' 0 ' and ' 1 ' in the codeword uneven. Hence the one-to-one non-linear mapping from 4-bit symbols to 6-bit symbols is designed, where the 4-bit symbol and the 6-bit symbol are represented as $s_{\mathrm{C}}$ and $s_{\mathrm{e}}$, 
respectively. Then, the decomposition means that each 6-bit symbol $s_{\mathrm{e}}$ is decomposed into two 3-bit sub-symbols, and sub-symbols with non-uniform probability distribution can be obtained. Finally, the sub-symbols are further permuted and recombined in pairs to generate the new 6-bit symbols $s_{\mathrm{p}}$ with more diverse probabilities, which are then mapped to generate the designed nonequiprobable constellation. Taking the probability distribution in Table 1 as the objective, the specific feasible example of non-linear mapping is presented as follows.

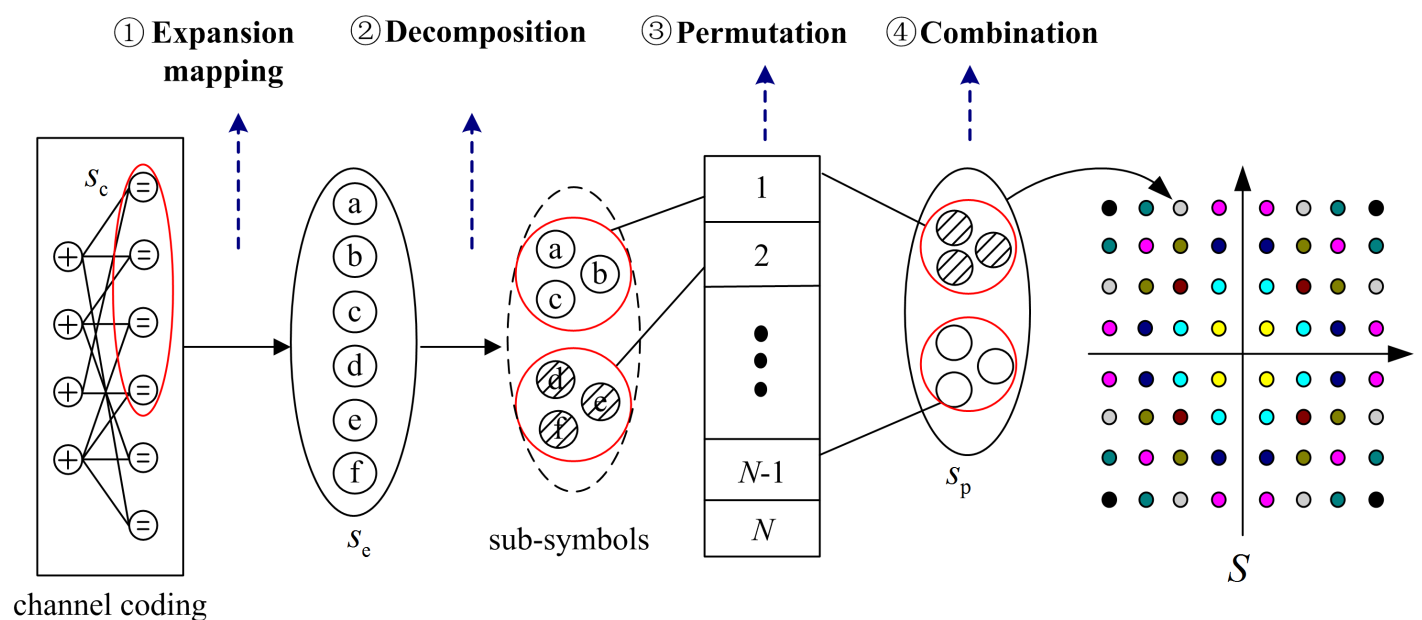

Figure 3. The process for generating nonequiprobable constellations.

First, we design the expansion mapping. $\chi_{m} \in\{0,1, \cdots, 63\}$ for $m=0,1, \cdots, 15$ is selected from all 6-bit symbols as the expansion mapping symbol set for one-to-one mapping. The expansion mapping relations from $s_{\mathrm{c}}$ to $s_{\mathrm{e}}$ are shown in Table 2 as an example, and the probability of each 4-bit symbol $s_{\mathrm{c}}$ and 6-bit symbol $s_{\mathrm{e}}$ is $1 / 16$. The design of expansion mapping is not unique, it only needs to satisfy that the sub-symbols after performing the next symbol decomposition step can conform to a certain non-uniform distribution.

Table 2. Expansion mapping rules.

\begin{tabular}{cccc}
\hline$s_{\mathbf{c}}$ & $s_{\mathbf{e}}$ & $s_{\mathbf{c}}$ & $\boldsymbol{s}_{\mathbf{e}}$ \\
\hline 0000 & 000000 & 1000 & 100000 \\
0001 & 000001 & 1001 & 100001 \\
0010 & 001011 & 1010 & 101011 \\
0011 & 000011 & 1011 & 100011 \\
0100 & 000100 & 1100 & 100100 \\
0101 & 011101 & 1101 & 111101 \\
0110 & 001111 & 1110 & 111110 \\
0111 & 011111 & 1111 & 111111 \\
\hline
\end{tabular}

Second, decompose the symbol $s_{\mathrm{e}}$. The symbol $s_{\mathrm{e}}$ is decomposed into 3-bit sub-symbols, denoted as $s_{\mathrm{e}}^{(i)}=\left(s_{\mathrm{L}}^{(i)}, s_{\mathrm{R}}^{(i)}\right)$, where sub-symbols $s_{\mathrm{L}}$ and $s_{\mathrm{R}}$ respectively correspond to the left and the right 3 bits of the 6-bit symbol $s_{\mathrm{e}}, i=0,1, \cdots, N-1$ and $N$ is the total number of symbols. Then the probability of sub-symbols is given by

$$
P\left(c_{n}\right)=\sum_{s_{\mathrm{L}}^{(i)}=c_{n} \text { or } s_{\mathrm{R}}^{(i)}=c_{n}} P\left(s_{\mathrm{e}}^{(i)}=\chi_{m}\right) / 2,
$$


where $c_{n}(n=0,1, \cdots, 7)$ represents the sub-symbol set, $\chi_{m}(m=0,1, \cdots, 15)$ represents the expansion symbol set, and $P\left(s_{\mathrm{e}}^{(i)}=\chi_{m}\right)=1 / 16$. After the 6-bit symbols $s_{\mathrm{e}}$ in the set $\chi_{m}$ are decomposed, for example, the sub-symbol ' 000 ' appears six times while the sub-symbol ' 010 ' does not appear, so their probability is 6/32 (0.1875) and 0, respectively. The probability of other sub-symbols can also be calculated. Thereby, the certain non-uniform probability distribution of sub-symbols shown in Table 3 can be obtained.

Table 3. Probability distribution of 3-bit sub-symbols.

\begin{tabular}{cc}
\hline Sub-Symbol & Probability \\
\hline 000 & 0.1875 \\
001 & 0.125 \\
010 & 0 \\
011 & 0.1875 \\
100 & 0.1875 \\
101 & 0.09375 \\
110 & 0.03125 \\
111 & 0.1875 \\
\hline
\end{tabular}

Third, perform the permutation, that is, the sub-symbols are randomly interleaved.

Finally, recombine the permuted sub-symbols in pairs to obtain nonequiprobable 6-bit mapping symbols $s_{\mathrm{p}}^{(j)}=\left(s_{\mathrm{L}}^{(j)}, s_{\mathrm{R}}^{(j)}\right)$.

Figure 4 illustrates the probability distribution of different symbol variables during the process of generating nonequiprobable symbol outputs. The symbol variables on the horizontal axis are expressed in decimal for convenience, and the probability corresponding to each symbol variable is given on the vertical axis. After the expansion mapping, the probability of each 6-bit symbol $s_{\mathrm{e}}$ selected into the expansion symbol set is the same as that of the 4-bit symbol $s_{c}$, which is $1 / 16$, and the probability of other unselected 6-bit symbols is 0 . Therefore, the expansion mapping step achieves a non-uniform probability distribution for the first time. Then, since the occurrence frequency of the sub-symbols is different in the designed expansion symbols $s_{\mathrm{e}}$, the non-uniformity of the probability for the sub-symbols is more obvious after the symbol decomposition. Furthermore, after the permutation and combination, symbols with more various probabilities can be obtained, which can be mapped to the signal points with different amplitudes shown in Table 1 to generate the nonequiprobable constellation.

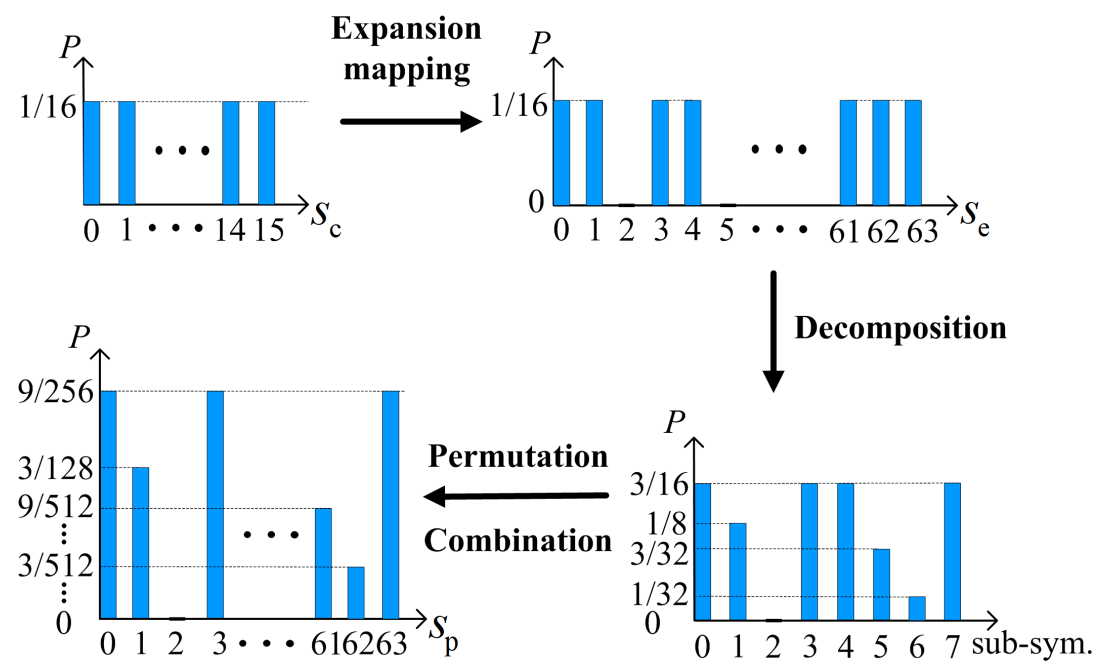

Figure 4. The probability distribution of the nonequiprobable symbols.

Figure 5 illustrates the three-dimensional diagram of the nonequiprobable 64-QAM constellation. The bottom surface represents the two-dimensional complex plane of the 64-QAM constellation, 
and the values on the vertical axis correspond to the probability of each constellation point. The left part in Figure 5 displays the constellation probability distribution of the design object shown in Table 1 , while the right one illustrates the probability distribution of the feasible example actually mapped by the nonequiprobable symbols, which are generated using the non-linear mapping method. It can be seen that the probability distribution proved by the feasible example is essentially consistent with the design object.
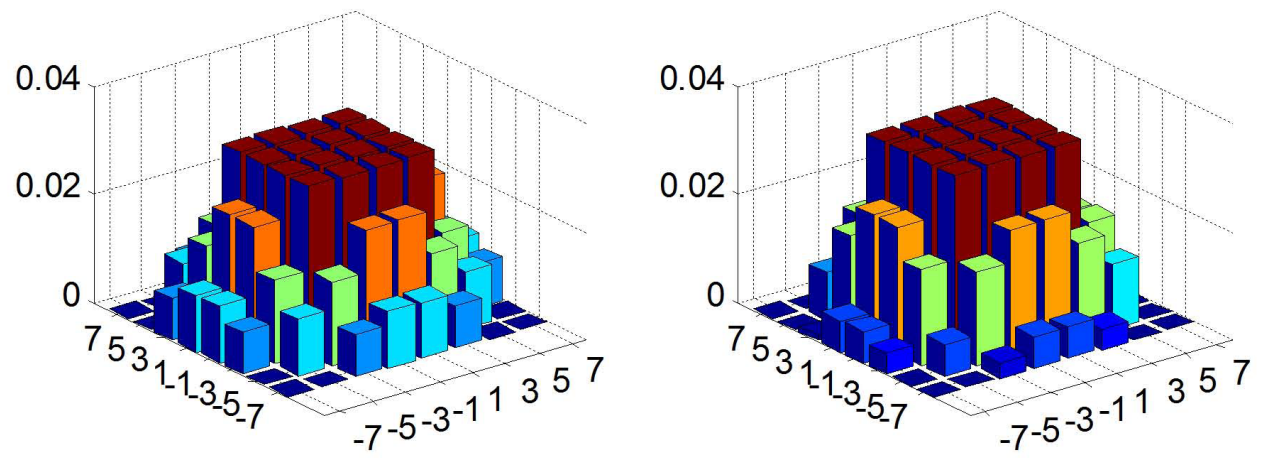

Figure 5. Probability distribution of the design object (left) and a feasible example (right).

\section{Demapping Method for the Nonequiprobable Constellation}

The estimated symbols associated to the transmitter are denoted as $\hat{s}_{\mathrm{p}}, \hat{s}_{\mathrm{L}}, \hat{s}_{\mathrm{R}}, \hat{s}_{\mathrm{e}}$, and $\hat{s}_{\mathrm{C}}$ at the receiver. The demapping method adapting to the proposed nonequiprobable constellation is illustrated in Figure 6.

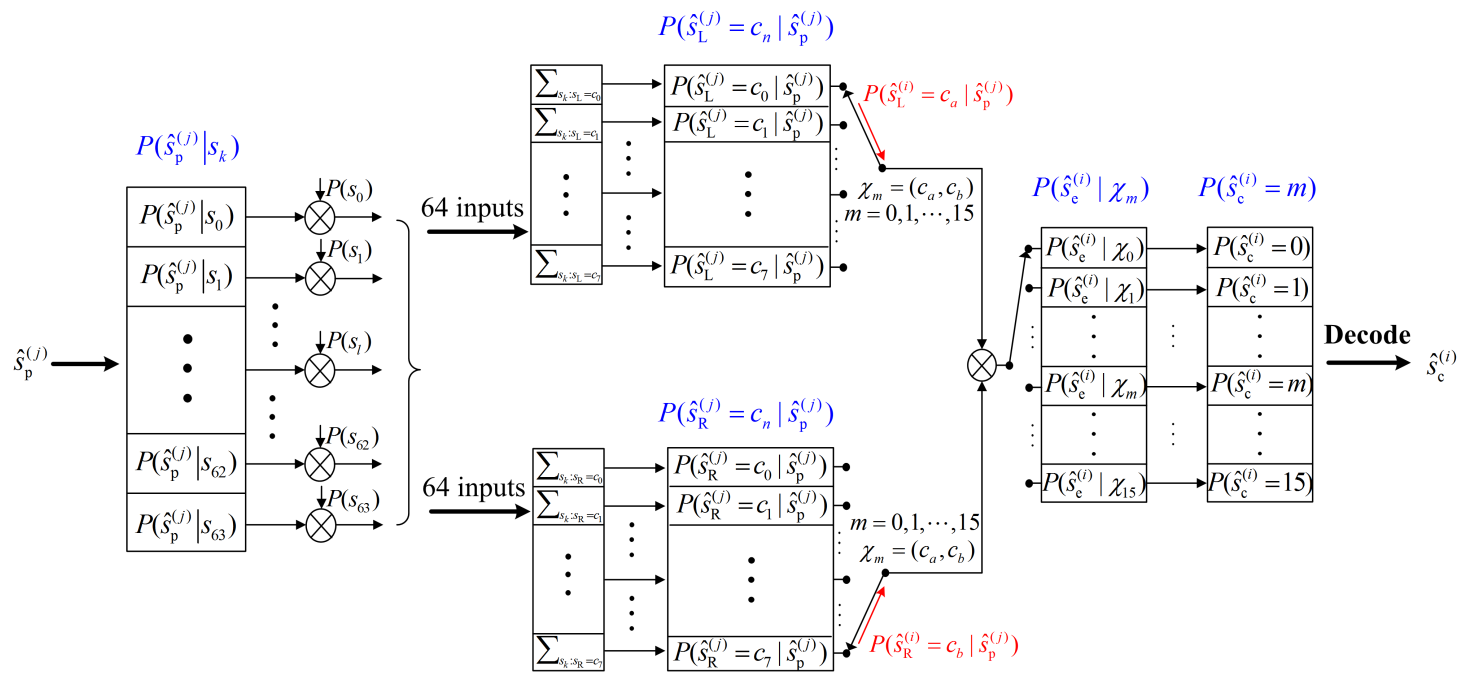

Figure 6. The demapping process for the nonequiprobable 64-QAM constellation.

First, according to the reference [20], since the Gaussian noise is added to the transmitted signal after non-linear conversions, the channel transition probability can be expressed as

$$
P\left(\hat{s}_{\mathrm{p}}^{(j)} \mid s_{k}\right)=\frac{1}{\pi N_{0}} \exp \left(\frac{-\left|\hat{s}_{\mathrm{p}}^{(j)}-s_{k}\right|^{2}}{N_{0}}\right),
$$

where $j=0,1, \cdots, N-1, s_{k}(k=0,1, \cdots, 63)$ is the distorted signal point on the designed constellation, and $\hat{s}_{\mathrm{p}}^{(j)}=\left(\hat{s}_{\mathrm{L}}^{(j)}, \hat{s}_{\mathrm{R}}^{(j)}\right)$. 
Then, the likelihood value of the sub-symbol $\hat{s}_{\mathrm{L}}$ or $\hat{s}_{\mathrm{R}}$ can be calculated as

$$
P\left(\hat{s}_{\mathrm{L}}^{(j)}=c_{n} \mid \hat{s}_{\mathrm{p}}^{(j)}\right)=\frac{\sum_{s_{k}: s_{\mathrm{L}}=c_{n}} P\left(\hat{s}_{\mathrm{p}}^{(j)} \mid s_{k}\right) P\left(s_{k}\right)}{\sum_{n=0}^{7} \sum_{s_{k}: s_{\mathrm{L}}=c_{n}} P\left(\hat{s}_{\mathrm{p}}^{(j)} \mid s_{k}\right) P\left(s_{k}\right)},
$$

where $P\left(s_{k}\right)$ corresponds to the prior probability for signal points of the constructed nonequiprobable constellation. Similarly, the likelihood value of $\hat{s}_{R}^{(j)}$ can also be calculated by replacing $\hat{s}_{\mathrm{L}}^{(j)}$ with $\hat{s}_{R}^{(j)}$ in Equation (7). Although $P\left(s_{k}\right)$ exists in both the numerator and denominator terms of Equation (7), the prior probabilities of the signal points in the nonequiprobable 64-QAM constellation are different, so they cannot be omitted.

Next, after the inverse process of the permutation and combination at the transmitter, we calculate the likelihood of $\hat{s}_{\mathrm{e}}^{(i)}=\left(\hat{s}_{\mathrm{L}}^{(i)}, \hat{s}_{\mathrm{R}}^{(i)}\right)$ as

$$
P\left(\hat{s}_{\mathrm{e}}^{(i)} \mid \chi_{m}\right)=P\left(\hat{s}_{\mathrm{L}}^{(i)}=c_{a} \mid \hat{s}_{\mathrm{p}}^{(j)}\right) \cdot P\left(\hat{s}_{\mathrm{R}}^{(i)}=c_{b} \mid \hat{s}_{\mathrm{p}}^{(j)}\right),
$$

where $\chi_{m}=\left(c_{a}, c_{b}\right), m=0,1, \cdots, 15$ and $0 \leq a, b \leq 7$.

Finally, the symbol likelihood value of $\hat{s}_{\mathrm{c}}^{(i)}$ can be obtained by one-to-one expansion demapping, which is denoted as

$$
P\left(\hat{s}_{\mathrm{c}}^{(i)}=m\right)=P\left(\hat{s}_{\mathbf{e}}^{(i)} \mid \chi_{m}\right),
$$

where $m=0,1, \cdots, 15$ is the decimal form of the 4-bit symbol $s_{\mathrm{c}}$. The symbol likelihood can be used directly or converted to the bit likelihood for decoding. The demapping method is summarized in Algorithm 1.

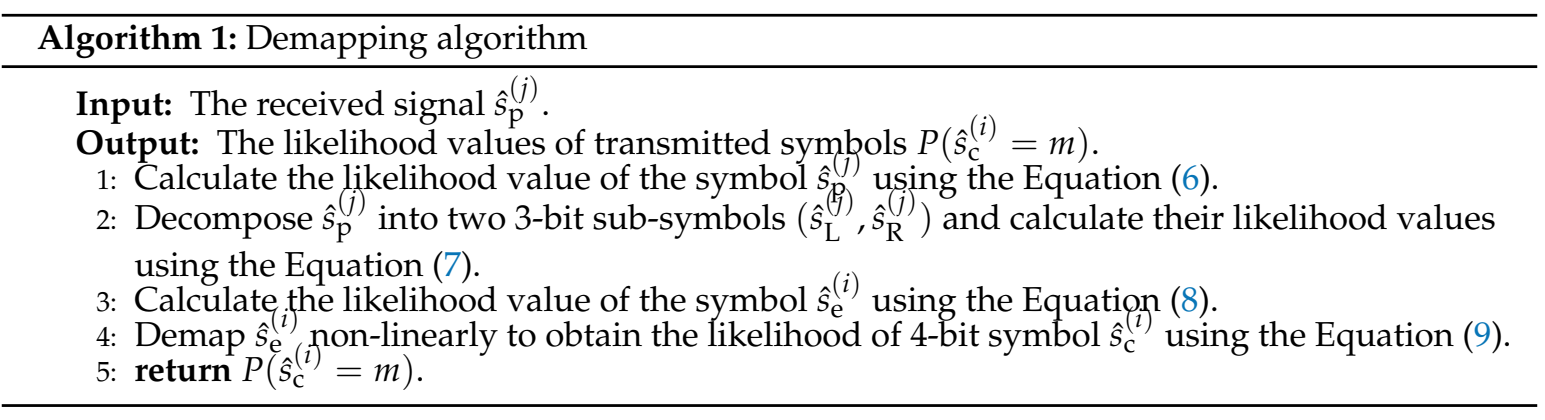

\section{Simulation Results}

This section investigates the bit error rate (BER) comparison of the proposed nonequiprobable constellation, conventional equiprobable 64-QAM constellation and 64-level amplitude phase shift keying (APSK) constellation which has four concentric rings and each ring consists of 16 points [35]. We use the low-density parity-check (LDPC) code with equiprobable 64-QAM or 64-APSK signal sets, and the non-binary LDPC (NB-LDPC) code over the Galois Field GF(16) with a nonequiprobable signal set. In this way, the symbol likelihood from demapping can be directly used to decode the NB-LDPC code. The non-linear mapping of the nonequiprobable signal set has a mapping from 4-bit to 6-bit, which is equivalent to occupying a code rate of $2 / 3$. Therefore, in order to compare fairly, the rates of the LDPC codes used for equiprobable and nonequiprobable signal sets are set to $1 / 2$ and 3/4, respectively; hence, their equivalent code rates are both $1 / 2$. The code length of the LDPC code is 2304 bits and the code length of the NB-LDPC code is 576 symbols, also 2304 bits. The parity check matrix of NB-LDPC codes has a row weight of 8 and a column weight of 2, and the non-zero elements in each row are chosen randomly from the set $\{1,2, \cdots, 15\}$ over $\mathrm{GF}(16)$. 
Figure 7 shows the BER comparison of the proposed nonequiprobable (nep) 64-QAM constellation, traditional equiprobable (ep) 64-QAM and 64-APSK constellations over the non-linear channel at different IBOs of $0 \mathrm{~dB}, 3 \mathrm{~dB}$, and $6 \mathrm{~dB}$. When the IBO is $0 \mathrm{~dB}$ or $3 \mathrm{~dB}$, the power amplifier is considered to work in or around the non-linear region. Considering that the nonequiprobable 64-QAM constellation is less influenced by the non-linear distortion while the performance of the equiprobable constellations deteriorates greatly, its overall performance is superior to the traditional equiprobable constellations. As shown in Figure 7, when the IBO is $3 \mathrm{~dB}$, the proposed nonequiprobable constellation gains 0.55 $\mathrm{dB}$ and $0.2 \mathrm{~dB}$ compared to the equiprobable 64-QAM and 64-APSK constellations at BER $=10^{-5}$. When the IBO is $0 \mathrm{~dB}$, we can even achieve improvement of $0.75 \mathrm{~dB}$ and $0.25 \mathrm{~dB}$, respectively, which is attributed to the better adaptation to the non-linear distortion of the proposed nonequiprobable constellation. When the IBO is $6 \mathrm{~dB}$, we observed that the proposed scheme does not work well.

On the one hand, although the equivalent code rates are the same, the $(4,6)$ non-linear mapping of the nonequiprobable signal set is designed for the non-uniform probability but could not be explicitly designed for error correction, so its overall error correction performance may be worse than the equiprobable signal set. On the other hand, the power amplifier at this time is considered to work close to the linear region, so the advantage of the nonequiprobable constellation for adapting to the non-linear distortion is less obvious. Hence the proposed scheme does not perform well at large IBOs, and the same is true for linear channels, as shown in Figure 8. In general, the proposed nonequiprobable constellation is especially applicable for scenarios with extremely severe non-linear distortion.

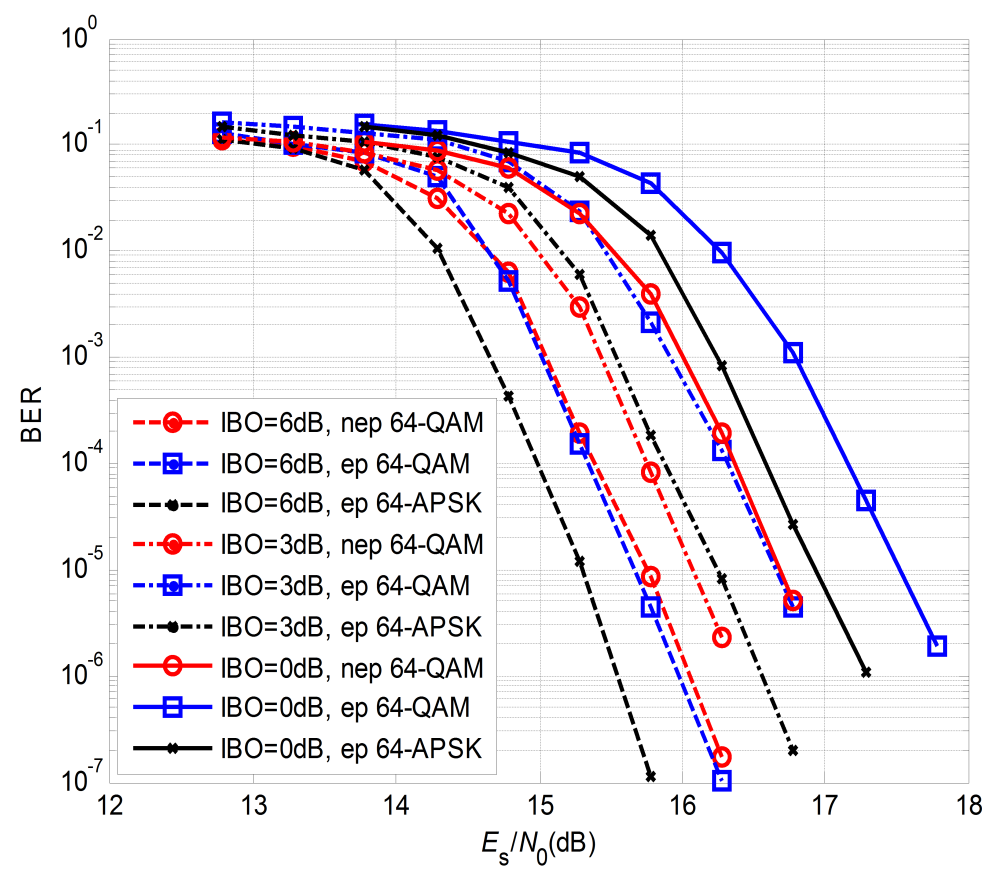

Figure 7. Bit error rate (BER) performance of different constellations over the non-linear channel at different IBOs. 


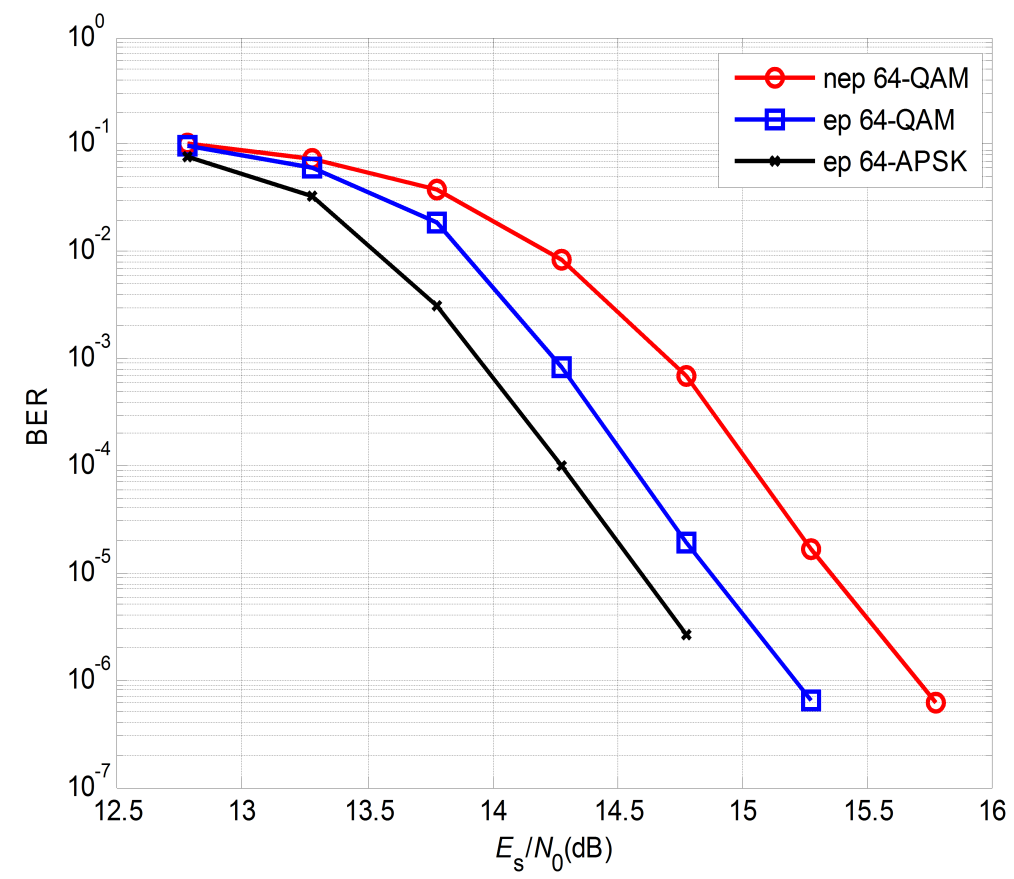

Figure 8. BER performance of different constellations over the linear channel.

\section{Conclusions}

In this paper, we designed a nonequiprobable constellation adapting to the non-linear distortion characteristics of the high power amplifier in satellite channels. First, we introduced a non-linear mapping method to generate the non-uniformly distributed symbol outputs, which specifically included expansion mapping, symbol decomposition, permutation, and combination, and used a one-to-one mapping to construct the target nonequiprobable constellation. Then, we provided the demapping method corresponding to the designed nonequiprobable constellation at the receiver. The simulation results illustrate that the designed nonequiprobable constellation is particularly suitable for scenarios with extremely severe non-linear distortion, and that it can effectively enhance the overall BER performance by adapting to the non-linear effects.

Author Contributions: Conceptualization, W.C. and Y.P.; methodology, W.C., C.H. and J.Y.; software, Y.P.; formal analysis, W.C. and Y.P.; investigation, W.C. and Y.P.; writing-original draft preparation, Y.P.; writing-review and editing, W.C., C.H. and J.Y. All authors have read and agreed to the published version of the manuscript.

Funding: This research was funded in part by the National Natural Science Foundation of China grant No. 61671324 and the Director's Funding from the Pilot National Laboratory for Marine Science and Technology (Qingdao).

Conflicts of Interest: The authors declare no conflict of interest.

\section{References}

1. Lansing, F.; Lemmerman, L.; Walton, A.; Bothwell, G.; Bhasin, K.; Prescott, G. Needs for communications and onboard processing in the vision era. In Proceedings of the IEEE International Geoscience and Remote Sensing Symposium, Toronto, ON, Canada, 24-28 June 2002; pp. 375-377.

2. Landon, D.; Simons, R.; Wintucky, E.; Sun, J.; Winn, J.; Laraway, A.; McIntire, W.; Mets, J.; Smith, F. Peak satellite-to-earth data rates derived from measurements of a $20 \mathrm{Gbps}$ bread-board modem. In Proceedings of the 2011 Military Communications Conference (MILCOM), Baltimore, MD, USA, 7-10 November 2011; pp. 1958-1963.

3. Simons, R.N. Link analysis of high-throughput spacecraft communication systems for future science missions antenna applications corner. IEEE Antennas Propag. Mag. 2016, 58, 66-73. [CrossRef] 
4. Benedetto,S.; Garello, R.; Montorsi, G.; Berrou, C.; Douillard, C.; Giancristofaro, D.; Ginesi, A.; Giugno, L.; Luise, M. MHOMS: High-speed ACM modem for satellite applications. IEEE Wirel. Commun. 2005, 12, 66-77. [CrossRef]

5. Morello, A.; Mignone, V. DVB-S2: The second generation standard for satellite broad-band services. Proc. IEEE 2006, 94, 210-227. [CrossRef]

6. Casini, E.; De Gaudenzi, R.; Ginesi, A. DVB-S2 modem algorithms design and performance over typical satellite channels. Int. J. Satell. Commun. Netw. 2004, 22, 281-318. [CrossRef]

7. Layton, K.J.; Mehboob, A.; Akhlaq, A.; Bagaglini, F.; Cowley, W.G.; Lechner, G. Predistortion for wideband nonlinear satellite downlinks. IEEE Commun. Lett. 2017, 21, 1985-1988. [CrossRef]

8. Xue, R.; Yu, H.; Cheng, Q. Adaptive coded modulation based on continuous phase modulation for inter-satellite links of global navigation satellite systems. IEEE Access 2018, 6, 20652-20662. [CrossRef]

9. Sung, W.; Kang, S.; Kim, P.; Chang, D.; Shin, D. Performance analysis of APSK modulation for DVB-S2 transmission over nonlinear channels. Int. J. Satell. Commun. Netw. 2009, 27, 295-311. [CrossRef]

10. De Gaudenzi, R.; Guillen i Fabregas, A.; Martinez, A. Performance analysis of turbo-coded APSK modulations over nonlinear satellite channels. IEEE Trans. Wirel. Commun. 2006, 5, 2396-2407. [CrossRef]

11. Singya, P.K.; Kumar, N.; Bhatia, V.; Alouini, M. On performance of hexagonal, cross, and rectangular QAM for multi-relay systems. IEEE Access 2019, 7, 60602-60616. [CrossRef]

12. Anedda, M.; Meloni, A. 64-APSK constellation and mapping optimization for satellite broadcasting using genetic algorithms. IEEE Trans. Broadcast. 2016, 62, 1-9. [CrossRef]

13. Yoda, D.; Ochiai, H. A reduced-complexity multilevel coded modulation for APSK signaling. In Proceedings of the 2013 IEEE International Symposium on Information Theory, Istanbul, Turkey, 7-12 July 2013; pp. 1994-1998.

14. Cronie, H.S. Signal shaping for bit-interleaved coded modulation on the AWGN channel. IEEE Trans. Commun. 2010, 58, 3428-3435. [CrossRef]

15. Agrell, E.; Alvarado, A. Signal shaping for BICM at low SNR. IEEE Trans. Inf. Theory 2013, 59, $2396-2410$. [CrossRef]

16. Sadeghi, A.; Lahouti, F.; Zorzi, M. Constellation shaping and LDPC coding in a bidirectional full duplex communication. In Proceedings of the 2015 IEEE Global Communications Conference (GLOBECOM), San Diego, CA, USA, 6-10 December 2015; pp. 1-6.

17. Yang, M.; Rastegarfar, H.; Djordjevic, I.B. Probabilistically coded modulation formats for 5G mobile fronthaul networks. J. Light. Technol. 2019, 37, 3882-3892. [CrossRef]

18. Ma, X.; Ping, L. Coded modulation using superimposed binary codes. IEEE Trans. Inf. Theory 2004, 50, 3331-3343. [CrossRef]

19. Bocherer, G.; Steiner, F.; Schulte, P. Bandwidth effificient and rate-matched low-density parity-check coded modulation. IEEE Trans. Commun. 2015, 63, 4651-4665. [CrossRef]

20. Ngo, N.H.; Barbulescu, S.A.; Pietrobon, S.S. Performance of nonuniform M-ary QAM constellation on nonlinear channels. In Proceedings of the 2005 Australian Communications Theory Workshop, Brisbane, Australia, 2-4 February 2005; pp. 79-83.

21. Yankov, M.; Forchhammer, S.; Larsen, K.J.; Christensen, L.P.B. Rate-adaptive constellation shaping for near-capacity achieving turbo coded BICM. In Proceedings of the 2014 IEEE International Conference on Communications (ICC), Sydney, Australia, 10-14 June 2014; pp. 2112-2117.

22. Valenti, M.C.; Xiang, X. Constellation shaping for bit-interleaved LDPC coded APSK. IEEE Trans. Commun. 2012, 60, 2960-2970. [CrossRef]

23. Burnet, C.E.; Ngo, N.H.; Pietrobon, S.S.; Cowley, W.G. Combined data predistortion and nonuniform distribution for 16QAM over the nonlinear satellite channel. In Proceedings of the 4th International Symposium on Turbo Codes \& Related Topics; 6th International ITG-Conference on Source and Channel Coding, Munich, Germany, 3-7 April 2006; pp. 1-6.

24. Kayhan, F; Montorsi, G. Constellation design for transmission over nonlinear satellite channels. In Proceedings of the 2012 IEEE Global Communications Conference (GLOBECOM), Anaheim, CA, USA, 3-7 December 2012; pp. 3401-3406.

25. Liu, Y.; Chen, W.; Wang, A.; Han, C. Combination of high-order modulation and non-binary LDPC codes over GF(7) for non-linear satellite channels. Electronics 2019, 8, 1396. [CrossRef] 
26. Saleh, A.A.M. Frequency-independent and frequency-dependent nonlinear models of TWT amplifiers. IEEE Trans. Commun. 1981, 29, 1715-1720. [CrossRef]

27. Meloni, A.; Murroni, M. On the genetic optimization of APSK constellations for satellite broadcasting. In Proceedings of the 2014 IEEE International Symposium on Broadband Multimedia Systems and Broadcasting, Beijing, China, 25-27 June 2014; pp. 1-6.

28. Long, Z.; Wu, N.; Wang, H.; Guo, Q. Turbo equalization based on a combined VMP-BP algorithm for nonlinear satellite channels. IEEE Access 2018, 6, 35492-35500. [CrossRef]

29. Yu, C.; Ibnkahla, M. Exact symbol error rate and total degradation performance of nonlinear M-QAM fading channels. In Proceedings of the IEEE International Conference on Acoustics, Speech, and Signal Processing (ICASSP), Philadelphia, PA, USA, 23 March 2005; pp. 985-988.

30. Song, L.; Wang, J.; Pan, C.; Fu, J. A normalized LLR soft information demapping method in DTMB system. In Proceedings of the 2008 11th IEEE Singapore International Conference on Communication Systems, Guangzhou, China, 19-21 November 2008; pp. 1297-1301.

31. Han, G.; Guan, Y.L.; Huang, X. Check node reliability-based scheduling for BP decoding of non-binary LDPC codes. IEEE Trans. Commun. 2013, 61, 877-885. [CrossRef]

32. Hu, H.; Yankov, M.P.; Da Ros, F.; Amma, Y.; Sasaki, Y.; Mizuno, T.; Miyamoto, Y.; Galili, M.; Forchhammer, S.; Oxenløwe, L.K.; Morioka, T. Ultrahigh-spectral-efficiency WDM/SDM transmission using PDM-1024-QAM probabilistic shaping with adaptive rate. J. Light. Technol. 2018, 36, 1304-1308. [CrossRef]

33. Dimitrov, S. Non-Linear distortion cancellation and symbol-based equalization in satellite forward links. IEEE Trans. Wirel. Commun. 2017, 16, 4489-4502. [CrossRef]

34. Ortega, A.; Fabbri, L.; Tralli, V. Performance evaluation of GFDM over nonlinear channel. In Proceedings of the 2016 International Conference on Information and Communication Technology Convergence (ICTC), Jeju, Korea, 19-21 October 2016; pp. 12-17.

35. Digital Video Broadcasting (DVB). Second Generation Framing Structure, Channel Coding and Modulation Systems for Broadcasting, Interactive Services, New Gathering and Other Broadband Satellite Applications; Part2: DVB-S2 Extensions (DVB-S2X); ETSI EN 302 307-2 V1.1.1, 2015. Available online: http://www. etsi.org/deliver/etsi_en/302300_302399/30230702/01.01.01_60/en_30230702v010101p.pdf (accessed on 8 January 2020).

(C) 2020 by the authors. Licensee MDPI, Basel, Switzerland. This article is an open access article distributed under the terms and conditions of the Creative Commons Attribution (CC BY) license (http:/ / creativecommons.org/licenses/by/4.0/). 